${ }^{3}$ National Institute of Public Health, University of Southern Denmark, Copenhagen, Denmark

\subsection{6/oemed-2013-101717.295}

Objectives To assess ergonomic exposures in a historical cohort study on musculoskeletal disorders among baggage handlers using production statistics from the airport and the involved handling company.

In historical cohort studies information on exposures is typically based on self-reports or assumptions of previous exposures. This may lead to misclassification of cumulative exposures. We analysed production data from the participating companies to improve the exposure assessments of the individual workers.

Methods Data from 1990-2012 regarding the number of flight operations was provided by Copenhagen Airport. The handling company provided data from 1998-2012 on total baggage weight, number of operations and number of baggage handlers on duty. Additionally, information existed back to 2002 on type of aircrafts, percentage of wide-bodied aircrafts (where some of the baggage was loaded in containers) and weight of manually handled baggage. It was possible to receive information about the dimension of the baggage compartments, which is decisive for the postures adopted in the hold (standing, stooping, squatting, kneeling and sitting). Information also existed on the time of introduction of technical lifting gear.

Results Although the number of passengers almost doubled during the past 20 years, the number of flight operations decreased as the number of passengers per flight increased. However, the weight of manually handled luggage out on the ramp decreased, probably because the number of wide-bodied aircrafts increased. Seasonal and daily variations existed. Because of the detailed production statistics it was possible to create a job exposure matrix by job tasks and calendar periods. This information will be combined with individual information on job tasks back in time.

Conclusion Although production statistics cannot provide information on individual factors such as the worker's lifting technique and his use of lifting gear, production statistics may be a valuable tool in exposure assessments in epidemiological studies.

\section{INFLUENCE FROM NON-OCCUPATIONAL FACTORS ON SELF-REPORTED OCCUPATIONAL LIFTING AMONG PREGNANT WOMEN: FINDINGS FROM THE DANISH NATIONAL BIRTH COHORT}

${ }^{1} \mathrm{P}$ F Frost, ${ }^{2}$ Svendsen, ${ }^{3}$ Mocevic, ${ }^{3}$ Jørgensen, ${ }^{4}$ Nybo Andersen, ${ }^{3}$ Bonde. ${ }^{1}$ Aarhus University Hospital, Aarhus C, Denmark; 'Herning Regional Hospital, Herning, Denmark; ${ }^{3}$ Bispebjerg University Hospital, Copenhagen, Denmark; ${ }^{4}$ University of Copenhagen, Copenhagen, Denmark

\subsection{6/oemed-2013-101717.296}

Objective To establish exposure estimates based on prospective interviews in a cohort of pregnant women using a job and industry group-based approach, and to evaluate whether non-occupational factors should be considered when a group-based strategy is chosen based on self-report.

Methods Within the Danish National Birth Cohort, a job exposure matrix (JEM) based on information from participants still pregnant at date of interview had been constructed. Job titles were coded using the Danish version of the International Standard Classification of Occupations (DISCO-88) and the Danish
Industrial Classification of All Economic Activities of employment industry was obtained from Statistics Denmark. We calculated the mean number of kilograms lifted per day within groups defined firstly by identical four digits DISCO- 88 codes and employment industry, secondly by identical four, three or two digits DISCO-88 codes, depending on a compulsory group size of $\geq 10$ participants. Level of self reported occupational lifting in relation to JEM-estimate and non-occupational factors including gestational age at interview, mother's age, parity, smoking and body mass index (bmi) was analysed with restriction to participants with identical job and industry codes. We used multivariate regression with bootstrap to obtain regression coefficients and 95\% confidence intervals.

Results A total of 41,405 women were included in the analyses. Gestational age $>97$ days at interview and increasing mother's age were associated with reporting of lower levels of occupational lifting, whereas bmi $\geq 30 \mathrm{~kg} / \mathrm{m}^{2}$, smoking while pregnant, and being a mother was associated with reporting of higher levels. Differences ranged from $-15 \mathrm{~kg}$ to $35 \mathrm{~kg}$.

Conclusion We constructed a JEM based on self-report to obtain group-based exposure estimates, striving to minimise information bias and attenuation in exposure response analyses. Non-occupational factors influence reporting of occupational lifting among pregnant women. This should be considered when a group-based strategy is used for exposure assessment based on self-report.

\section{GROUP-BASED EXPOSURE MEASUREMENT STRATEGIES AND THEIR EFFECTS ON TRUNK ROTATION AND LOW- BACK PAIN EXPOSURE-OUTCOME ASSOCIATIONS}

${ }^{1} \mathrm{P}$ Coenen, ${ }^{2}$ Mathiassen, ${ }^{3}$ Kingma, ${ }^{4}$ Boot, ${ }^{5}$ Bongers, ${ }^{3}$ van Van Dieen. ${ }^{1}$ Vrije Universiteit, Amsterdam, The Netherlands; '2University of Gavle, Gavle, Sweden; ${ }^{3} \mathrm{VU}$ University, Amsterdam, Nederland; ${ }^{4}$ VU Medical Center, Amsterdam, Nederland; ${ }^{5}$ TNO Healthy Living, Hoofddorp, Nederland

\subsection{6/oemed-2013-101717.297}

Objectives In epidemiological studies of occupational exposures (e.g. lifting) and low-back pain (LBP), group-based exposure measurement strategies are common. Workers are classified into exposure groups; exposure is measured only in a selection of workers in each group, and their mean exposure is assigned to all workers in the group. Exposure-outcome relationships are then determined by regression, relating exposure estimates with individual LBP data from all subjects. The objective of this study was to assess the effect of different group-based measurement strategies on exposure-outcome associations.

Methods 1122 workers, classified into 19 groups on the basis of job-related exposure, participated in this study. In each group, videos were collected from $\sim 25 \%$ of the workers (in total, 370 workers), and percentage of the work day spent in trunk rotation was estimated by observation of the videos. This estimate of trunk rotation was significantly associated with self-reported LBP during three years of follow-up (OR:1.43 (1.06-1.93)).

Using a bootstrap simulation, workers per group $(n=10,20$, $30,40)$ and percentage of observed workers $(\mathrm{k}=10,20,30,40$, $50 \%$ ) were varied. For each combination, (nk) workers were selected with replacement in each job group among those observed, and n (100-k) workers among those not observed. The mean exposure of the observed workers was assigned to all group members which was related to individual LBP data. ORs and accompanying p-level was estimated using logistic-regression.

Results A group-based measurement protocol led to significant $(\mathrm{p}<0.05)$ ORs when the total number of workers was larger 